\title{
Possibility of using superoxide dismutase and glutathione peroxidase as endometriosis biomarkers
}

This article was published in the following Dove Press journal:

International Journal of Women's Health

27 September 2017

Number of times this article has been viewed

\section{Sophapun Ekarattanawong' Chamnan Tanprasertkul ${ }^{2}$ Charintip Somprasit ${ }^{2}$ Pholasit Chamod' \\ Rattana Tiengtip' \\ Kornkarn \\ Bhamarapravatana' Komsun Suwannarurk ${ }^{2}$}

'Department of Preclinical Science, ${ }^{2}$ Department of Obstetrics and Gynaecology, Faculty of Medicine, Thammasat University, Pathum Thani, Thailand
Correspondence: Sophapun

Ekarattanawong

Department of Preclinical Science, Faculty of Medicine, Thammasat University, 99 Paholyothin Road, Klong Luang, Pathum Thani 12120, Thailand

Tel +6629269710

Fax +66292607II

Email sophapun@hotmail.com
Objective: To study the possibility of using superoxide dismutase (SOD) and glutathione peroxidase (GPx) as combined preoperative biomarkers for endometriosis.

Participants and methods: Female patients aged between 18 and 45 years old who came to the Gynecology outpatient clinic, Thammasat University Hospital, during September 20132016 with the complaint of gynecologic symptoms suspected of endometriosis, and who were positively diagnosed with endometriosis, were included in this study. All patients underwent conservative laparoscopic ovarian cystectomy with histopathological report. The control group consisted of healthy females of reproductive age who came to the outpatient clinic. The plasma SOD and GPx were measured from blood samples with commercial kits. A receiver operating characteristic curve was generated for plasma activity of SOD, GPx, and combined tests. The cutoff values were selected at the most appropriate sensitivity and specificity.

Result: All 36 cases were included in this study. Mean ages of patients in the patient and control groups were 33.1 and 28.6 years old, respectively. SOD and GPx activities of disease and control group were 6.15 and $8.11,463.9$ and $472.34 \mathrm{nmole} / \mathrm{min} / \mathrm{mL}$ unit, respectively. The sensitivity and 1 -specificity of the combined test were calculated at 0.78 , with sensitivity, specificity, accuracy, positive predictive value, and negative predictive value being $68.75,80.77,76.50$, 69.23, and 80.33, respectively.

Conclusion: A combination testing of SOD and GPx can possibly be used as preoperative biomarker for endometriosis.

Keywords: superoxide dismutase, SOD, glutathione peroxidase, GPx, endometriosis, laparoscopy, endometriosis biomarker

\section{Introduction}

Endometriosis is a chronic gynecological disorder in females characterized by the displacement of endometrial glands and stroma in the pelvic peritoneal cavity. It is not only associated with infertility, but typically transforms into endometriotic cyst. ${ }^{1}$ Endometriosis increases reproductive health risk factors, ie, shorter menstrual cycle length, longer duration of flow, or reduced parity. It causes several symptoms including acute and chronic pelvic pain, dysmenorrhea, dyspareunia, abnormal vaginal bleeding, infertility/sterility, and, in severe stages, gastrointestinal and urological symptoms. ${ }^{2}$ Chronic nonmenstrual pain, dyspareunia, and infertility are the main common symptoms that cause a severe impact on patient's psychological well-being and quality of life, which are the reasons that bring patients to gynecologic consultation. ${ }^{3,4}$

Sampson's 5 implantation theory in 1927 demonstrated retrograde menstruation as the pathogenesis of endometriosis. However, Samson's theory did not explain cases of 
retrograde menstruation with no endometriosis $(90 \%$ of the condition) nor cases of endometriotic foci distant from the pelvis. It also did not explain the cases of endometriosis in male patients. The effect of endometriosis was additionally explained by immunological alterations of the peritoneal fluid, such as the inhibition of the physiological processes of cytolysis. Coelomic metaplasia and Müllerian remnants theories have later been proposed. ${ }^{6}$ Endometriosis has also been defined as a hormonal disease, an autoimmune disease, a/an (epi)genetic disorder, or due to environmental stimuli or nutritional deficiency. ${ }^{7}$

The endometriotic cyst is one of the endometriosis variations. It is composed of endometrial gland and stroma. It forms the pseudocapsule. Endometriotic cyst (the older term was endometriosis externa) is said to resemble ovarian cancer. They both have similar characters, ie, bilateral existence, peritoneal seeding, metastasis-like behavior, and CA125 elevation. The preoperative evaluation of endometriotic cyst consists of complete history taking, basic laboratory examination, thoroughly physical examination, pelvic examination, and imaging study (ultrasonography, computerized tomography, and magnetic resonance imaging). These preoperative evaluations are also similar to that carried out for ovarian cancer evaluation. ${ }^{1}$ In minimal severity cases, the surgeon prefers laparoscopic surgery. In the cases where diseases are of increased severity, the surgeons favor assigned exploratory laparotomy.

The gold standard for endometriosis diagnosis is a visual inspection by laparoscopy or laparotomy, followed by a histological confirmation. ${ }^{1}$ Due to the nature of invasive surgery coupled with the lack of a laboratory biomarker for the disease, both laparoscopy and laparotomy result in a delayed definitive diagnosis, thus leading to disease progression over time. A noninvasive diagnosis for endometriosis is highly desirable. Such a diagnosis might be able to rule out some patients from the gold standard surgery for endometriosisassociated pain and/or infertility. The definitive treatment of endometriosis is hysterectomy with oophorectomy. This treatment will make the patient enter into a menopausal state. They have to face menopausal symptoms prematurely.

Angiogenesis plays a vital role in endometriosis progression. ${ }^{2}$ miR-199a-5p regulates cell proliferation, motility, and angiogenesis through its effect on vascular endothelial growth factor A (VEGFA). It was found that VEGFA was overexpressed in ectopic endometriosis tissue. Serum analysis of miR-199a-5p was shown to be inversely correlated with VEGFA concentration. Functional analysis of miR199a-5p suggested that miR-199a-5p may represent both a diagnostic marker and a therapeutic target in the context of endometriosis. However, to date, there is no proposed cutoff value for miR-199a-5p.,

More than 130 articles from MEDLINE related to oxidative stress and antioxidants during 1990-2014 revealed that the imbalance between the formation of oxidants and the availability of endogenous antioxidants are the major determinant of endometriosis. ${ }^{10}$ Localized pelvic inflammatory reactions resulted in increased concentration of cytokines, growth factors, and other proinflammatory mediators such as hemoglobin and heme from erythrocytes. Oxidative stress localized to the peritoneal environment may be one of the links in the chain of events leading to endometriosis. ${ }^{11}$

There are many antioxidant enzymes such as superoxide dismutase (SOD), catalase, glutathione peroxidase (GPx), glutathione reductase (GR), and glutathione transferase. SOD is the first line of defense against oxygen free radicals. It catalyzes dismutation of superoxide anion radical into hydrogen peroxide $\left(\mathrm{H}_{2} \mathrm{O}_{2}\right)$. It could protect cells and extracellular components from cellular damage. However, SOD does not have a widespread usage in human clinical medicine. ${ }^{12}$

GPx, a major peroxide-scavenging enzyme, is one of the antioxidant enzyme for sequential elimination of superoxide radical and hydrogen peroxide. It prevents the formation of an extremely toxic hydroxyl radical. GPx also reduces lipid or nonlipid hydroperoxides while oxidizing glutathione (GSH). The oxidized GSH is then reduced by GR.

Currently, there is no validated biomarker(s) for any noninvasive diagnostic test for endometriosis possessing sufficient sensitivity and specificity. Biomarker research in endometriosis needs reproducible data with high sensitivity and specificity. The discovery of a specific biomarker with a high sensitivity for nonsurgical endometriosis detection would promise an earlier diagnosis and prevention of deleterious sequelae. Our investigation proposed the use of two antioxidant enzyme plasma activities, namely, SOD and GPx, as biomarkers for endometriosis.

\section{Participants and methods Subjects}

The protocol for the entire investigation was approved by the Human Research Ethic Committee of Thammasat University (COA 097/2556).

The subjects were female patients aged between 18-45 years old who came to Gynecology outpatient clinic, Thammasat University Hospital, during September 2013 to September 2016 with the complaint of gynecologic symptoms predictive of endometriosis. Those with positive 
endometriosis diagnosis by laparoscopy were recruited. They all signed the consent forms to be enrolled in the study. The exclusion criteria were alcohol consumption, consumption of vitamins or food supplements with antioxidants, report of infertility, and the use of contraceptives.

All participants in this study underwent conservative laparoscopic ovarian cystectomy. The time of surgery was during the follicular phase of menstrual cycle (no more than 14 days after subject's last menstrual period). The pneumoperitoneum creation was done using Veress needle insertion and carbon dioxide insufflation. The $5 \mathrm{~mm}$ diameter of trocar was punctured using the conventional method. Bipolar electrocautery was applied to cut and coagulate vessels during surgery. The final diagnosis was confirmed with a histopathological report.

The control group consisted of healthy females aged 18-45 years who came to gynecology walk-in outpatient clinic with no complaint of endometriosis or its related symptoms. The inclusion criteria were possessing anormal menstrual cycle. Exclusion criteria were the same as that of the study group.

A total of 36 subjects were enrolled in this study, 16 in the study group and 20 in the control group.

\section{Blood samples}

Two blood samples were taken from all participants before their laparoscopic ovarian cystectomy operations. The control group had one blood draw at the time of their outpatient visit. The protocol for blood sample collection was approved by Human Research Ethic Committee of Thammasat University.

Blood sample was drawn from the subject and placed in EDTA tube (Vacuette, Greiner Bio-One, Kremsmünster, Austria). Both SOD and GPx activities were measured using commercially available assay kits (Cayman Chemical, Ann Arbor, MI, USA) following the methodology described by the manufacturer. The blood sample was immediately placed on ice and transferred to the laboratory. Upon arrival, all samples were immediately ultracentrifuged for $10 \mathrm{~min}$ (Thermo Sorvall, Thermo Fisher Scientific, Waltham, MA, USA) at $1,000 \times g$ and $4^{\circ} \mathrm{C}$. The serum aliquot was obtained and kept frozen at $-80^{\circ} \mathrm{C}$ until further use for the plasma enzyme activity assay.

SOD activity in plasma was measured using a tetrazolium salt for detection of superoxide radicals generated by xanthine oxidase and hypoxanthine. The chromophore produced has a maximal absorbance at $525 \mathrm{~nm}$. One unit of SOD is defined as the amount of enzyme needed to exhibit $50 \%$ dismutation of the superoxide radical.
GPx activity was measured using a kinetic colorimetric assay that measured activity indirectly by a coupled reaction with GR. GR and NADPH reduce oxidized glutathione. NADPH oxidation is accompanied by a decrease in absorbance at $340 \mathrm{~nm}$. The decrease is directly proportional to the GPx activity in the sample.

A receiver operating characteristic curve (ROC) curve was generated for plasma activity of SOD and GPx using STATA version 13 (StataCorp LLC, College Station, TX, USA). The cutoff values then were selected at the most appropriated sensitivity and specificity. The areas under the curve between the two tests were compared. Finally, the two tests were combined and then the ROC curve was generated.

\section{Results}

During the recruitment period, 16 and 20 cases were enrolled into the study and control group, respectively. Members of the study group underwent laparoscopic surgery. All were diagnosed with endometriosis and were subjected to laparoscopic cystectomy. Demographic data and enzymatic activities of study and control groups are shown in Table 1. The average age of the study and control groups was 33 and 28 years, respectively. SOD activity was statistically significant at $p<0.05$. However, GPx activity showed no statistical significance.

SOD and GPx activity data from the study are presented in Table 1. The relationship between sensitivity and 1-specificity for SOD and GPx were assessed to find the ROC pattern of each enzyme (Figure 1). Areas under the ROC pattern for SOD and GPx were 0.7308 and 0.5288 , respectively. GPx activity showed less potency in this test compared to that of SOD.

The results of the measurement of the combined activities of SOD and GPx in the diagnosis of endometriosis are shown in Figure 2. When the plot was made, 0.78 was the chosen cutoff point as none of the tests had higher enzymatic activity

Table I Demographic data and average plasma SOD and GPx activity in the study population

\begin{tabular}{|c|c|c|}
\hline & $\begin{array}{l}\text { Control } \\
(n=25)\end{array}$ & $\begin{array}{l}\text { Study } \\
(n=16)\end{array}$ \\
\hline Age (years) ${ }^{\mathrm{a}}$ & $28.65 \pm 1.1$ & $33.13 \pm 1.17$ \\
\hline Parity & 1.2 & 0.2 \\
\hline SOD activity ${ }^{b}$ & $6.15 \pm 1.95$ & $8.11 \pm 0.62$ \\
\hline GPx activity ${ }^{\mathrm{b}}$ & $463.9 \pm 32.7$ & $472.34 \pm 43.17$ \\
\hline
\end{tabular}

Notes: a Mean \pm SD; ${ }^{b}$ Enzyme activities in $\mathrm{nmol} / \mathrm{min} / \mathrm{mL}$ unit; Control: healthy participants; Study; endometriosis patients.

Abbreviations: SOD, superoxide dismutase; GPx, glutathione peroxidase; SD, standard deviation. 


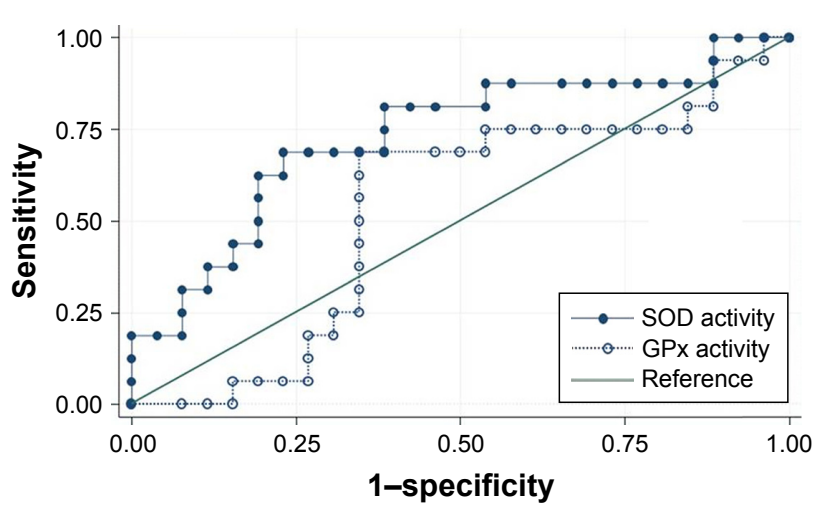

Figure I Summary ROC plot of superoxide dismutase; SOD (dark) and GPx (light) activity measurement in the diagnosis of endometriosis.

Note: SOD under curve area $=0.7398, \mathrm{GPx}$ under curve area $=0.5288$.

Abbreviations: ROC, receiver operating characteristic; SOD, superoxide dismutase; GPx, glutathione peroxidase.

than this one. It is also the appropriate number that gave the best sensitivity, specificity accuracy, positive predictive value (PPV), and negative predictive value (NPV).

The sensitivity and 1-specificity were then calculated for the approximate cutoff point. The predictive power of SOD, GPx, and the combined test are shown in Table 2. The combined test has sensitivity, specificity, accuracy, PPV, and NPV of $68.75,80.77,76.50,69.23$, and 80.33, respectively.

\section{Discussion}

The most common symptoms of endometriosis are chronic pelvic pain, severe dysmenorrhea, dyspareunia, and infertility. ${ }^{2-4}$ The final diagnosis of endometriosis is done by a laparoscopic procedure for visual inspection and based on the histology report. To date, there is no appropriate biomarker for endometriosis.

Biomarkers have been widely used in gynecology. Serum CA-125 was the most common tumor marker used

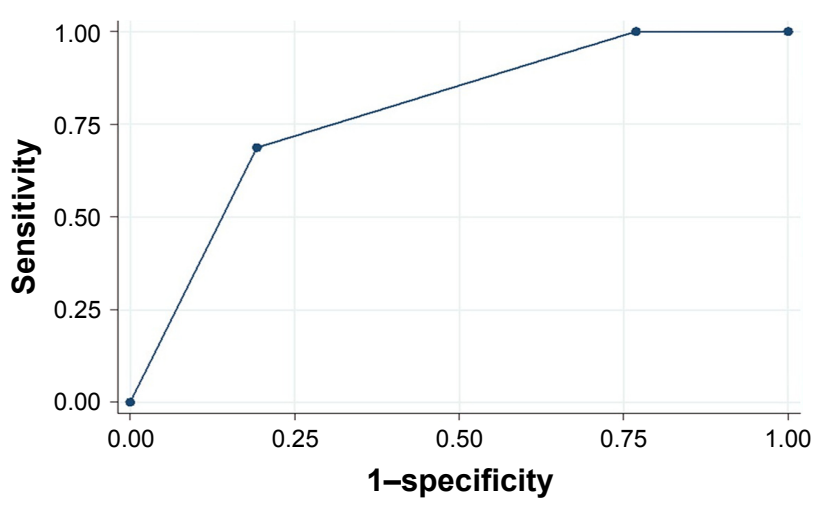

Figure 2 A combined ROC curve of combination detection serum SOD and GPx. Note: Area under ROC curve $=0.7837$.

Abbreviations: ROC, receiver operating characteristic; SOD, superoxide dismutase; GPx, glutathione peroxidase.
Table 2 Predictive power of enzyme activity of SOD, GPx, and of the combined test

\begin{tabular}{llllll}
\hline $\begin{array}{l}\text { Enzyme } \\
\text { system }\end{array}$ & Sensitivity & Specificity & Accuracy & PPV & NPV \\
\hline SOD & 81.00 & 57.69 & 67.45 & 55.17 & 83.33 \\
GPx & 75.00 & 46.50 & 57.35 & 46.77 & 73.68 \\
Combined & 68.75 & 80.77 & 76.50 & 69.23 & 80.33 \\
\hline
\end{tabular}

Abbreviations: SOD, superoxide dismutase; GPx, glutathione peroxidase; PPV, positive predictive value; NPV, negative predictive value.

in assessment of follow-up after gynecological cancer treatment. ${ }^{1}$ Serum concentration of CA-125 usually rises significantly in ovarian cancer patients. It can also rise in noncancer condition such as tubo-ovarian abscess. Endometriosis and other benign tumors also cause increase in serum CA-125. It is a nonspecific biomarker in gynecological investigations.

The attempt to use blood biomarkers for endometriosis has been investigated since $1998 .{ }^{13}$ However, the lack of specificity and low sensitivity of the studied biomarkers prevented further development. The use of CA 19-9, an ovarian tumor marker, has been attempted for detecting endometriosis. However, it had lower sensitivity compared to CA-125 in ovarian cancer patients. ${ }^{14}$ Serum miR-199a-5p, a regulator for VEGFA, was suggested for use as an endometriosis biomarker. ${ }^{8,9}$ However, there is no specificity and sensitivity study yet reported for miR-199a-5p.

It has been confirmed that many oxidative stress-related proteins, namely, paraoxonase-1, high density lipoproteins, SOD, and GPx show blood concentration changes in correlation with the endometriosis diagnosis. ${ }^{15,16}$ This is due to the retrograde flow of menstrual blood into the abdomen, resulting in accumulation of ruptured erythrocytes (Sampson's theory of reflux). Iron from ruptured erythrocytes causes a rise in oxidative stress. SOD activity was found to be lower in the blood of patients with leiomyoma, endometrial polyps, and in hyperplastic and malignant endometrium lipid peroxidation than in healthy subjects. ${ }^{17}$ The increased systemic oxidative stress in disease progression and its association with other oxidative stress-related pathologic conditions were suggested for further studies. ${ }^{18}$ No investigators in oxidative stress and disease progression reported the ROC curve analysis of any antioxidant enzymes for application in endometriosis diagnosis.

In clinical settings, when a patient came to the hospital with a complaint of pelvic pain, dyspareunia, or infertility, the provisional diagnosis was usually endometriosis. It means that there is an indication for a surgery based on the subjective data. When the patient chooses to undergo endometriosis treatment, the procedure will be a minimal invasive surgery as described for our study group. In patients who have symptoms identifiable with pelvic mass, either laparoscopy or exploratory 
laparotomy would be performed based on the attending physician's decision. The decision for surgery is not subjective. Laparoscopy would be conducted if any cysts were observed. The cyst could then be removed while in laparoscopic process. Cases with laparoscopy could also switched over to exploratory laparotomy intraoperatively.

From this study, the authors suggested the cutoff value for combined SOD and GPx activity. The sensitivity, specificity, accuracy, PPV, and NPV were 68.75, 80.77, 76.50, 69.23, and 80.33 , respectively. A biomarker assay with a fair PPV would be a fresh welcome to endometriosis screening. This investigation opens the door to further research and development of combined SOD and GPx tests for endometriosis screening.

Preoperative biomarker testing is a much less invasive test to detect endometriosis in patients compared to the current gold standard technique. It also costs less than the cost of minimal laparoscopic surgery procedure $(2,000$ USD in government hospital). The cost of the enzymatic activities per sample will decrease when the testing market size is increased. The current drawback of this combined test is that it uses a 96-well system (Cayman Chemical). There is a need to develop the test into a radioimmunoassay strip for each test to be performed individually.

In many occasions, preoperative techniques for endometriosis detection, such as ultrasound, cannot reveal pathologic findings. These SOD and GPx tests could be used for preoperative check in patients who have symptom of chronic pelvic pain, progressive dysmenorrheal, and severe dyspareunia. The possible use of endometriosis biomarkers might help rule out patients who come in for infertility complaint but do not suffer endometriosis as the pathological cause (about half of the cases of infertility are associated with endometriosis). ${ }^{19}$ The SOD and GPx enzyme activity may be able to give positive supportive evidence to the surgeon, thereby allowing an objective tipping point for an exploratory laparotomy. The possible use of noninvasive biomarkers for endometriosis screening might prompt more women to come for early investigation and shorten the long-term psychological effects associated with the disease.

\section{Acknowledgment}

The authors gratefully acknowledge the financial support provided by Thammasat University under the TU Research scholar grant, contract number TP2/49/2556.

\section{Disclosure}

The authors report no conflicts of interest in this work.

\section{References}

1. Berek JS, Longacre TA, Friedlander M. Ovarían, fallopian tube, and peritoneal cáncer. In: Berek JS, editor. Berek \& Novak's Gynecology. 15th ed. New York, NY: Lippincott Williams \& Wilkins; 2012: 1350-1428.

2. D'Hooghe TM. Endometriosis. In: Berek JS, editor. Berek \& Novak's Gynecology. 15th ed. New York, NY: Lippincott Williams \& Wilkins; 2012:505-556.

3. Laganà AS, La Rosa VL, Rapisarda AMC, et al. Anxiety and depression in patients with endometriosis: impact and management challenges. Int $J$ Womens Health. 2017;9:323-330.

4. Soliman AM, Coyne KS, Zaiser E, Castelli-Haley J, Fuldeore MJ. The burden of endometriosis symptoms on health-related quality of life in women in the United States: a cross-sectional study. $J$ Psychosom Obstet Gynaecol. 2017;21:1-11.

5. Sampson JA. Metastatic or embolic endometriosis, due to the menstrual dissemination of endometrial tissue into the venous circulation. Am J Pathol. 1927;3(2):93-110.

6. Uğur M, Turan C, Mungan T, et al. Endometriosis in association with müllerian anomalies. Gynecol Obstet Invest. 1995;40(4):261-264.

7. Laganà AS, Vitale SG, Salmeri FM, et al. Unus pro omnibus, omnes pro uno: a novel, evidence-based, unifying theory for the pathogenesis of endometriosis. Med Hypotheses. 2017;103:10-20.

8. Liu XJ, Bai XG, Teng YL, Song L, Lu N, Yang RQ. miRNA-15a-5p regulates VEGFA in endometrial mesenchymal stem cells and contributes to the pathogenesis of endometriosis. Eur Rev Med Pharmacol Sci. 2016;20(16):3319-3326.

9. Chia-Yi H, Tsung-Hua H, Cheng-Fang T, et al. miRNA-199a-5p regulates VEGFA in endometrial mesenchymal stem cells and contributes to the pathogenesis of endometriosis. J Pathol. 2014;232(3): 330-343.

10. Iwabuchi T, Yoshimoto C, Shigetomi H, Kobayashi H. Oxidative stress and antioxidant defense in endometriosis and its malignant transformation. Oxid Med Cell Longev. 2015;2015:848595.

11. Gupta S, Agarwal A, Krajcir N, Alvarez JG. Role of oxidative stress in endometriosis. Reprod Biomed Online. 2006;13(1):126-134.

12. McCord JM, Edeas MA. SOD, oxidative stress and human pathologies: a brief history and a future vision. Biomed Pharmacother. 2005;59(4): 139-142.

13. Fassbender A, Burney RO, Dorien FO, D’Hooghe T, Giudice L. Update on biomarkers for the detection of endometriosis. Biomed Res Int 2015;2015:130854.

14. Vodolazkaia A, El-Alamat Y, Popovic D, et al. Evaluation of a panel of 28 biomarkers for the non-invasive diagnosis of endometriosis. Hum Reprod. 2012;27(9):2698-2711.

15. Verit FF, Erel O, Celik N. Serum paraoxonase-1 activity in women with endometriosis and its relationship with the stage of the disease. Hum Reprod. 2008;23(1):100-104.

16. Prieto L, Quesada JF, Cambero O, et al. Analysis of follicular fluid and serum markers of oxidative stress in women with infertility related to endometriosis. Fertil Steril. 2012;98(1):126-130.

17. Pejić Snezana, Todorović A, Stojiljković V, Kasapović J, Pajović SB Antioxidant enzymes and lipid peroxidation in endometrium of patients with polyps, myoma, hyperplasia and adenocarcinoma. Reprod Biol Endocrinol. 2009;7.

18. Lambrinoudaki IV, Augoulea A, Christodoulakos GE, et al. Measurable serum markers of oxidative stress response in women with endometriosis. Fertil Steril. 2009;91(1):46-50.

19. Bulletti C, Coccia ME, Battistoni S, Borini A. Endometriosis and infertility. J Assist Reprod Genet. 2010;27(8):441-447. 
International Journal of Women's Health

Dovepress

\section{Publish your work in this journal}

The International Journal of Women's Health is an international, peerreviewed open-access journal publishing original research, reports, editorials, reviews and commentaries on all aspects of women's healthcare including gynecology, obstetrics, and breast cancer. The manuscript management system is completely online and includes Visit http://www.dovepress.com/testimonials.php to read real quotes from published authors.

Submit your manuscript here: http://www.dovepress.com/international-journal-of-womens-health-journal 